\title{
MELHORIAS EM PROCESSOS COM IMPACTO NA EFICIÊNCIA OPERACIONAL: UM ESTUDO AMBIENTADO EM UM LABORATÓRIO DE ANÁLISES CLÍNICAS
}

\section{PROCESS IMPROVEMENT WITH IMPACT IN THE OPERATIONAL EFFICIENCY: A STUDY IN THE CONTEXT OF A CLINICAL ANALYSIS LABORATORY}

\author{
Gabriel Sperandio Milan* E-mail: gsmilan@ucs.br \\ Roberta Versetti* E-mail: roberta.versetti@gmail.com \\ *Universidade de Caxias do Sul (UCS), Caxias do Sul, RS
}

Resumo: Em um ambiente de intensa competição e mudanças constantes, caracterizadas por exigências cada vez maiores em termos de rapidez, qualidade, baixo custo, flexibilidade e satisfação do cliente, o sucesso de uma organização da área da saúde reside na sua capacidade de responder adequadamente a estas exigências por meio de inovações em seus serviços e processos. Neste contexto, como objetivo central do trabalho, destaca-se a identificação de uma alternativa para a qualificação da operação de um Laboratório de Análises Clínicas, a partir de seus processos internos. Para isso, o trabalho utiliza o mapeamento de processos como base de análise e proposição. Sendo assim, foram analisados os macroprocessos do Laboratório e identificadas oportunidades de melhoria. Além disso, foi proposta a reestruturação dos macroprocessos analisados, apresentando os recursos necessários à implementação da proposta, bem como indicadores de desempenho e suas respectivas metas.

Palavras-chave: Gestão por Processos. Processos em Serviços. Eficiência Operacional. Serviços de Saúde. Laboratório de Análises Clínicas.

Abstract: In an environment of constant change and intense competition, characterized by increasing demands in terms of speed, quality, low cost, flexibility and customer satisfaction, the success of a healthcare organization is its ability to respond appropriately to these requirements by means innovations in its services and processes. In this context, the central objective of the study is identifying an alternative to qualify the Clinical Analysis Laboratory operation from its internal processes. For this, the paper uses process mapping as a basis for analysis and proposition. Therefore, we analyzed the macro process of the Laboratory and identified opportunities for improvement. Moreover, it was proposed the restructuring of the macro process analyzed, presenting the resources needed to implement the proposal as well as the performance indicators and their targets.

Keywords: Process Management. Processes in Services. Operational Efficiency. Health Services. Clinical Analysis Laboratory.

\section{INTRODUÇÃO}

As constantes mudanças tecnológicas e a inserção das organizações em um ambiente de competição globalizada tornam necessário que se (re)projete a 
operação e a gestão das funções organizacionais, de modo a adequá-las à realidade do mercado e às expectativas dos clientes, visando aumentar a competitividade e o valor da organização (VILELLA, 2000). Diante deste cenário, uma das principais funções do gestor, inclusive na área da saúde, é a busca por soluções que permitam o aperfeiçoamento constante dos processos de trabalho e a oferta de serviços diferenciados. Sendo assim, a gestão por processos proporciona a definição da melhor maneira e sequência para a realização de cada atividade do processo em si (mapeamento dos processos) e dos indicadores gerenciais que possibilitarão a medição, análise e melhoria de cada processo (MELLO et al., 2002).

Para tanto, o mapeamento dos processos de uma organização pode contribuir para uma melhor compreensão do funcionamento interno e dos relacionamentos entre os processos empresariais (HARRINGTON, 1993). De acordo com Johnston e Clark (2002), o principal benefício obtido pelo mapeamento dos processos de serviços é o surgimento de uma visão e de um entendimento compartilhado de um processo por todos os envolvidos e, assim, uma realização de seu papel no processo de serviço do princípio ao fim.

Neste contexto, é oportuno comentar que o mercado de análises clínicas, no Brasil, está passando por profundas transformações. A evolução tecnológica tem trazido grandes avanços, porém, as grandes dificuldades em se manter atualizado estão, principalmente, nos altos custos da tecnologia e os baixos valores pagos pelos serviços prestados. As perspectivas para o setor são no sentido de que quem acompanhar toda esta evolução alinhada à capacidade em gestão, continuará no mercado, pois aqueles que não evoluírem, não sobreviverão.

\section{REFERENCIAL TEÓRICO}

\subsection{Gestão por Processos}

Todo e qualquer serviço prestado a um cliente necessita de um processo para sua realização. A concepção mais frequente de processo é um grupo de atividades interligadas e realizadas em uma sequência lógica, que utiliza os recursos da organização para gerar os resultados definidos e com o objetivo de gerar um serviço 
que tenha valor para os clientes (HARRINGTON, 1993; HAMMER; CHAMPY, 1994; JOHANSSON et al., 1995) e que os satisfaça (MILAN; TREZ, 2005). Para Villela (2000), um processo deve dispor de entradas (insumos), saídas (resultados), tempo, espaço, ordenação, objetivos e valores que, interligados logicamente, irão resultar em uma estrutura para fornecer produtos ou serviços ao cliente, sendo a sua compreensão um fator essencial para o sucesso de qualquer negócio.

Portanto, o processo é um conceito fundamental no projeto dos meios pelos quais um provedor de serviços de saúde preste seus serviços aos seus clientes. Além disso, muitos dos processos nas empresas são repetitivos e envolvem, no seu conjunto, a maioria das pessoas da organização (GONÇALVES, 2000). Sendo assim, é imperativo que as organizações da área da saúde adotem meios adequados para gerenciar as interações entre os processos existentes.

Pode-se, portanto, definir a gestão por processos como uma metodologia para contínua avaliação, análise e melhoria do desempenho dos processos-chaves da organização, ou seja, os que mais impactam na satisfação dos clientes (MELLO et al., 2002). Além disso, a gestão por processos proporciona a definição da melhor maneira e sequência para a realização de cada atividade do processo (mapeamento dos processos) e dos indicadores gerenciais que possibilitarão a medição, análise e melhoria de cada processo. Inclusive, Johansson et al. (1995) defendem que uma organização é tão efetiva quanto forem os seus processos, pois eles são responsáveis pelo que será ofertado ao cliente.

Davenport (1995), por sua vez, conceitua a reengenharia de processos como uma alteração de processo que provoque uma mudança importante e radical, ou seja, esta técnica modifica a estrutura das atividades do processo, visando "resultados visíveis e drásticos". Sob esta ótica, o autor salienta que a reengenharia de processos se distingue da melhoria de processos, pois esta visa um nível mais superficial de mudanças. Segundo Hammer e Champy (1995), a reengenharia de processos de negócios significa uma reestruturação radical dos processos, com o objetivo de alcançar melhorias drásticas em indicadores de desempenho como custos, qualidade, atendimento ao cliente e velocidade.

Dos conceitos citados pelos autores, conclui-se que a reengenharia de processos de negócios é um esforço organizado para reestruturar integralmente os 
processos inerentes aos tipos de negócios de uma organização, visando proporcionar ganhos significativos em indicadores de desempenho, tais como: custos, qualidade, velocidade e satisfação do cliente, bem como a possibilidade de obtenção de uma vantagem competitiva. Os processos implantados por uma reengenharia são comprimidos horizontalmente, em que os empregados realizam mais tarefas sequenciais, trabalhando em processos mais abrangentes, e verticalmente, em que passam a tomar decisões antes delegadas apenas aos gerentes. Os benefícios destas "compressões" são: a redução na demora ou atrasos (na prestação de serviços ou em seus resultados), menores custos, melhor atendimento aos clientes e maior nível de delegação de poder (empowerment) aos colaboradores (HAMMER; CHAMPY, 1995).

Neste contexto, o mapeamento de processos é uma ferramenta gerencial analítica e de comunicação essencial para os gestores e organizações inovadoras na área da saúde que têm a intenção de promover melhorias ou implantar uma estrutura voltada para novos processos (VILELLA, 2000). Pode-se dizer, então, que o mapeamento de processos desempenha o papel essencial de desafiar os processos existentes, criando oportunidades de melhoria de desempenho organizacional ao identificar interfaces críticas e, sobretudo, criar bases para implantação de novas e modernas tecnologias de informação (TI) e de integração empresarial. Para Hunt (1996), esta análise estruturada de processos permite, ainda, reduzir custos no desenvolvimento novos serviços, falhas de integração entre sistemas e promover melhoria de desempenho organizacional (lucro, rentabilidade), além de ser uma excelente forma para o melhor entendimento dos processos atuais e para a eliminação ou a simplificação dos que necessitam de mudanças.

O mapeamento dos processos também ganha importância por sua função de registro e documentação histórica da organização. Uma vez que o aprendizado é construído com base em conhecimentos e experiências passadas dos indivíduos (conhecimento implícito ou tácito), a organização não pode se arriscar, em função de seus funcionários migrarem de um emprego para outro ou se aposentarem, a perder lições e experiências conseguidas ao longo de muitos anos (VILELLA, 2000).

No mapeamento de processos, consideram-se atividades, informações e restrições de interface de forma simultânea, com a sua representação iniciando-se a 
partir do sistema inteiro de processos, como uma única unidade modular que será expandida em diversas outras unidades mais detalhadas (subprocessos) que, por sua vez, serão decompostas em maiores detalhes de forma sucessiva. Tal decomposição é que garantirá a validade dos mapas finais (VILELLA, 2000).

Assim sendo, o mapa de processos deve ser apresentado sob a forma de uma linguagem gráfica, que permita expor os detalhes do processo de modo gradual e controlado; encorajar concisão e precisão na descrição do processo; focar a atenção nas interfaces do mapa do processo; e fornecer uma análise de processos consistente com o "vocabulário" do projeto (HUNT, 1996).

Por conseguinte, o mapeamento é realizado pela utilização de uma técnica para representar as diversas tarefas necessárias, na sequência em que elas ocorrem para a realização e entrega do serviço. Existem, na literatura, diversas técnicas de modelagem ou mapeamento de processos de negócios. A algumas possuem aplicação em quaisquer tipos de operações, inclusive em serviços (fluxograma tradicional, IDEF0), e outras especialmente desenvolvidas ou adaptadas para processos em serviços (service blueprint, mapas do serviço, walk-through-audit, análise da transação de serviço, IDEF3 adaptado, linguagem de representação para projeto de processos de serviços) (SANTOS; VARVAKIS, 2001).

\subsection{Gestão por Processos em Serviços}

No contexto específico dos serviços, os processos podem significar a sequência de atividades necessárias para conduzir as transações ocorridas na prestação do serviço (RAMASWAMY, 1996). Lovelock (1995) afirma que a melhoria da qualidade em serviços só é possível com profundo entendimento do processo. Assim, o processo assume uma importância primordial também na gestão de serviços, pois é ele o determinante da natureza das interações entre o usuário e a organização, conhecidas como "momentos da verdade".

Shostack (1984) foi um dos primeiros autores a introduzir na literatura o planejamento de serviços sob a perspectiva do processo. Esta autora desenvolveu a técnica de mapeamento de processos de serviços chamada Service Blueprint ou Blueprint do Serviço, que pode ser utilizada tanto no planejamento quanto na análise 
do processo (FITZSIMMONS; FITZSIMMONS, 2010). Esta característica é defendida por Gummesson (1994), para quem o planejamento e a análise de processos de serviços deveriam ser integrados por meio de uma única técnica.

O Service Blueprint, apesar de ser derivado dos fluxogramas tradicionais usados na gestão de processos industriais, diferencia-se destes, pois considera a interação com o usuário do serviço (FITZSIMMONS; FITZSIMMONS, 2010). Segundo Brown, Fisk e Bitner (1994), uma diferença primária entre o Blueprint e o fluxograma tradicional é que incorpora o cliente e as ações do cliente no mesmo fluxograma (mapa de contexto) que representa a operação ou o funcionamento do serviço. Ou seja, o processo é visto também sob a perspectiva do cliente.

De acordo com Fitzsimmons e Fitzsimmons (2010), portanto, o Blueprint é um mapa de todas as transações que constituem o processo de entrega do serviço. Este mapa identifica tanto as atividades da linha de frente (pessoal de contato direto) quanto às atividades da retaguarda (pessoal de suporte), separadas por uma linha de visibilidade, identificando o pessoal de linha de frente (front office) e o pessoal de retaguarda (back office). Consoante isso, Schmenner (1999) propõe a aplicação do Blueprint na identificação dos gargalos do processo de planejamento da capacidade de atendimento e na avaliação dos tempos de execução do serviço, na análise dos custos envolvidos, entre outros. Em acréscimo, Lovelock (1995) acrescenta a esta lista de aplicações o replanejamento do processo de trabalho. Já Gianesi e Corrêa (1994) analisam o Blueprint com a intenção de identificar os processos-chave, para em seguida estabelecer a relação destes com as áreas de decisão da empresa.

A partir do estudo do Blueprint de um serviço, é possível emergir oportunidades de melhorias e, também, a necessidade de uma melhor definição futura de certos processos. No caso de serviços, pode-se utilizar uma "linha de visibilidade", que separa as atividades do atendimento, onde os clientes obtêm evidências tangíveis do serviço, daquelas atividades de retaguarda, que não são vistas pelo cliente. Ademais, a utilização do Blueprint oferece aos gestores a oportunidade de identificar potenciais pontos de falha e projetar procedimentos à prova de erros (FITZSIMMONS; FITZSIMMONS, 2010).

De acordo com Johnston e Clark (2002), o principal benefício obtido pelo mapeamento dos processos de serviços, o que é premente na área da saúde, é o 
surgimento de uma visão e de um entendimento compartilhado de um processo por todos os envolvidos e, desta forma, uma realização de seu papel no processo de serviço do princípio ao fim.

\subsection{Eficiência Operacional}

O desenvolvimento de um processo gerencial que melhore requer, necessariamente, o empreendimento de um esforço sistemático para projetar, reprojetar e implantar sistemas de medição e avaliação de desempenho. Isso significa que a medição e a avaliação da eficiência, eficácia, efetividade, qualidade, produtividade, inovação, lucratividade etc. são parte integrante dos sistemas de suporte ao processo de gestão, que busca a competitividade. Sink e Tuttle (1993) salientam que o desempenho organizacional normalmente é analisado basicamente em função de três dimensões: eficiência, eficácia e efetividade.

Catelli (2001) afirma que a eficiência é o processo pelo qual a organização maximiza seus fins com uso mínimo de recursos. Eficiência diz respeito ao método, ao modo certo de fazer as coisas. É definida pela relação entre volumes produzidos/recursos consumidos. Uma organização eficiente é aquela que consegue o seu volume de produção com o menor dispêndio possível de recursos. A medida de eficiência pode ser representada por meio de indicador. O nível de eficiência apresentado por uma organização está relacionado com os recursos consumidos/utilizados e com os serviços prestados, considerando que tais recursos e serviços possuem valor econômico, expressos na forma de custos e receitas.

É por isso que os níveis de eficiência impactam os resultados econômicos de uma organização.

Entretanto, a eficácia reflete a qualidade e adaptabilidade dos serviços, à operação e/ou ao mercado, ou ainda quão bem as expectativas do cliente estão sendo atendidas frente aos seus requisitos técnicos. A eficácia pode ser medida por meio dos resultados alcançados pela organização frente ao que foi planejado (SILVA, 2007), ou seja, a eficácia organizacional é o alcance dos objetivos e das metas determinados e a correta determinação destes objetivos e metas (direcionadores). Já a efetividade, por sua vez, apresenta o conceito mais amplo que 
busca avaliar os resultados das ações implantadas, verificando os reais benefícios que estas ações trarão.

Nesta direção, emerge a necessidade premente de se entender melhor, principalmente, as dimensões de eficiência e de eficácia no contexto do desempenho dos serviços (TORRES JÚNIOR; MIYAKE, 2011). Para tanto, o uso de indicadores de desempenho para a aferição dos resultados de forma balanceada e associada aos objetivos e às metas estabelecidas é uma excelente ferramenta para monitoramento da eficácia dos processos, já que "o processo é controlado através dos seus efeitos" (CAMPOS, 1999, p. 19). A utilização de indicadores de desempenho está diretamente associada ao entendimento do processo por se tratar de uma fotografia da sua situação em um determinado momento. Aliás, como diz Hronec (1994), são os sinais vitais da organização. Sua principal função é manter o processo sob controle, o que, segundo Campos (1999), é possibilitar aos gestores saberem localizar o problema, analisar o processo, padronizar e estabelecer itens de controle de tal forma que o problema nunca mais ocorra.

De acordo com Takashima e Flores (1996), os indicadores proporcionam à gestão uma melhoria contínua da qualidade dos serviços e o aumento da produtividade da organização, ampliando a satisfação de seus clientes e melhorando a sua imagem, competitividade e participação no mercado. Eles quantificam as características de serviços e processos, sendo utilizados para controlar e melhorar a qualidade e o desempenho dos mesmos.

É imprescindível observar alguns critérios para a criação dos indicadores, destacando a relevância, simplicidade e clareza, abrangência, rastreabilidade e acessibilidade, comparabilidade, estabilidade e rapidez de disponibilidade e baixo custo de obtenção dos dados (MARTINS; NETO, 1998).

Após a definição dos indicadores, deve-se atribuir uma meta, a qual consiste na determinação de um valor pretendido ao indicador em determinadas condições. Esta meta deve estar relacionada diretamente as estratégias da organização. Para que a criação dos indicadores seja bem sucedida, faz-se necessário o desdobramento até o nível da estação de trabalho, visando proporcionar um maior controle no processo de acompanhamento das metas (MARTINS; NETO, 1998). 
No entanto, cabe aos gestores, de acordo com as peculiaridades de seu negócio, o que é bastante comum aos serviços de saúde, identificar os elementos de controle (instrumentos e indicadores) que possam atendê-los em suas medições, possibilitando um comparativo permanente em relação às metas e os padrões de desempenho previamente definidos.

\section{MÉTODO DE PESQUISA}

\subsection{Caracterização do Ambiente em Estudo}

O desenvolvimento do estudo foi ambientado junto a um Laboratório de Análises Clínicas de um Hospital-Escola localizado no interior do Estado do Rio Grande do Sul (RS), o qual é uma Instituição referência no atendimento do Sistema Único de Saúde (SUS) para a Serra Gaúcha e para a Região da $5^{\text {a }}$ Coordenadoria Regional de Saúde (CRS), que compreende, ao todo, 48 municípios.

O Laboratório de Análises Clínicas possui equipamentos de diagnósticos com tecnologia sofisticada para realização de exames clínicos nas áreas de bioquímica, hematologia, urinálise, imunologia, dosagens de hormônios, parasitologia, coagulação e bacteriologia, entre outros tipos mais complexos. O Hospital trata-se de uma instituição de grande porte, possuindo 267 leitos em funcionamento e está habilitado a realizar procedimentos de média e alta complexidade para pacientes ambulatoriais e internados. O Laboratório de Análises Clínicas, por sua vez, atende toda a demanda de exames necessários a estes pacientes, realizando mensalmente, em média, 4.000 atendimentos e 40.000 exames.

O processo analítico no laboratório clínico é composto de diferentes fases sucessivas denominadas: fase pré-analítica, analítica e pós-analítica. A fase préanalítica engloba as etapas desde a recepção do cliente na unidade de atendimento até o transporte do material para a área de processamento analítico. A fase analítica começa com a validação do sistema analítico através do controle da qualidade interno na amplitude normal e patológica e termina quando a determinação analítica gera um resultado. Por fim, a fase pós-analítica diz respeito à aprovação e liberação do resultado gerado na fase analítica, ou seja, a emissão do laudo por profissional 
habilitado. Vale ressaltar que a fase pré-analítica deve ter sua padronização criteriosamente definida e constantemente monitorada em razão do impacto significativo das atividades realizadas nesta fase do processo sobre a qualidade do resultado para as determinações laboratoriais processadas.

A partir desse ambiente de estudos, foram identificados e analisados os macroprocessos de atendimento ao cliente, coleta de amostras biológicas, triagem e segregação do material (fase pré-analítica) e aprovação dos resultados (fase pósanalítica).

\subsection{Objetivos do Estudo}

O objetivo geral do trabalho é identificar alternativa para a qualificação da operação do Laboratório de Análises Clínicas em estudo, a partir de seus processos internos. Além disso, foram estabelecidos os seguintes objetivos específicos: (i) analisar os macroprocessos do laboratório, do atendimento ao cliente à entrega dos resultados; (ii) identificar oportunidades de melhorias; (iii) elaborar uma proposta de reestruturação para os macroprocessos analisados; (iv) apresentar os recursos necessários à implantação da proposta; e (v) redefinir os principais indicadores de desempenho com suas respectivas metas.

\subsection{Técnicas e Procedimentos de Pesquisa}

Para Richardson (1999), a pesquisa qualitativa foca a experiência das pessoas e seu respectivo significado em relação a eventos, processos e estruturas, inseridos em cenários sociais. Consoante isso, foi desenvolvido um estudo qualitativo com procedimento técnico classificado como pesquisa participante, que se desenvolve a partir da interação entre pesquisadores e membros das situações investigadas (GIL, 2008), no caso, profissionais do Laboratório em estudo. $\mathrm{Na}$ observação participativa, o observador não é apenas um espectador do fato que está sendo estudado, ele se coloca na posição e ao nível dos outros elementos humanos que compõem o fenômeno a ser observado (RICHARDSON, 1999). 
É consenso entre os autores (BRANDÃO, 1990; GIL, 2008; RICHARDSON, 1999) que, concomitante com outros métodos, a pesquisa participante permite a reafirmação de fatos, facilitada pela vivência de situações específicas. $\mathrm{O}$ pesquisador sente intensamente as dificuldades e as facilidades das situações vivenciadas, inclusive as adaptações necessárias para compreender o campo de interrogativas, fruto de novas inquietações que emergem à medida que passa por essas vivências. Portanto, foi empregado, como método de levantamento de dados, a observação participativa, através da interação dos pesquisadores com os processos a serem analisados, como também a pesquisa documental, por meio da utilização de manuais, relatórios e documentos pertencentes aos processos selecionados.

Uma vez identificados os principais macroprocessos, e suas características, foi realizado o mapeamento destes processos, com o objetivo de melhor compreende-los e para identificar oportunidades de melhoria que embasassem a elaboração de uma proposta de reestruturação, apresentando os recursos necessários à sua implementação, além da redefinição dos principais indicadores de desempenho e metas para monitorá-los. Como comentado por Johansson et al. (1995) e por Fitzsimmons e Fitzsimmons (2010), a realização deste mapeamento possibilita a identificação das interfaces críticas, a identificação de oportunidades para simulações de processos, a implantação de métodos de gestão baseados em atividades e a identificação de pontos isolados ou incoerentes nos processos.

\section{DESENVOLVIMENTO DA PESQUISA}

\subsection{Caracterização e Mapeamento dos Macroprocessos em Estudo}

Para melhor entender os macroprocessos existentes no Laboratório, foi realizado o diagnóstico dos mesmos a fim de propiciar um melhor entendimento dos problemas existentes e garantir a melhoria do processo. Ao iniciar tal atividade, observou-se que não havia um controle sistemático dos processos, ou seja, apenas alguns processos estavam mapeados, não havendo ligação alguma entre os principais processos. Algumas atividades praticadas seguiam procedimentos informalmente estabelecidos, sem padronização, monitoramento e verificação 
periódica destas atividades. Para facilitar tal compreensão, a Figura 1, apresentada logo a seguir, é elucidativa.

Para melhor compreensão do processo de atendimento aos clientes existente, este foi dividido em processo ambulatorial e hospitalar, entendendo-se como processo ambulatorial, o atendimento de pacientes com exames de rotinas, sem a necessidade de emergência; enquanto 0 atendimento hospitalar se refere ao atendimento aos pacientes que estão internados no hospital, necessitando de prioridade com relação aos pacientes ambulatoriais. $O$ atendimento de urgência para pacientes internados deve ser imediato, sem protelação, devendo ser prestado em um período de tempo não superior a duas horas.

Figura 1 - Mapa de contexto atual dos macroprocessos do Laboratório
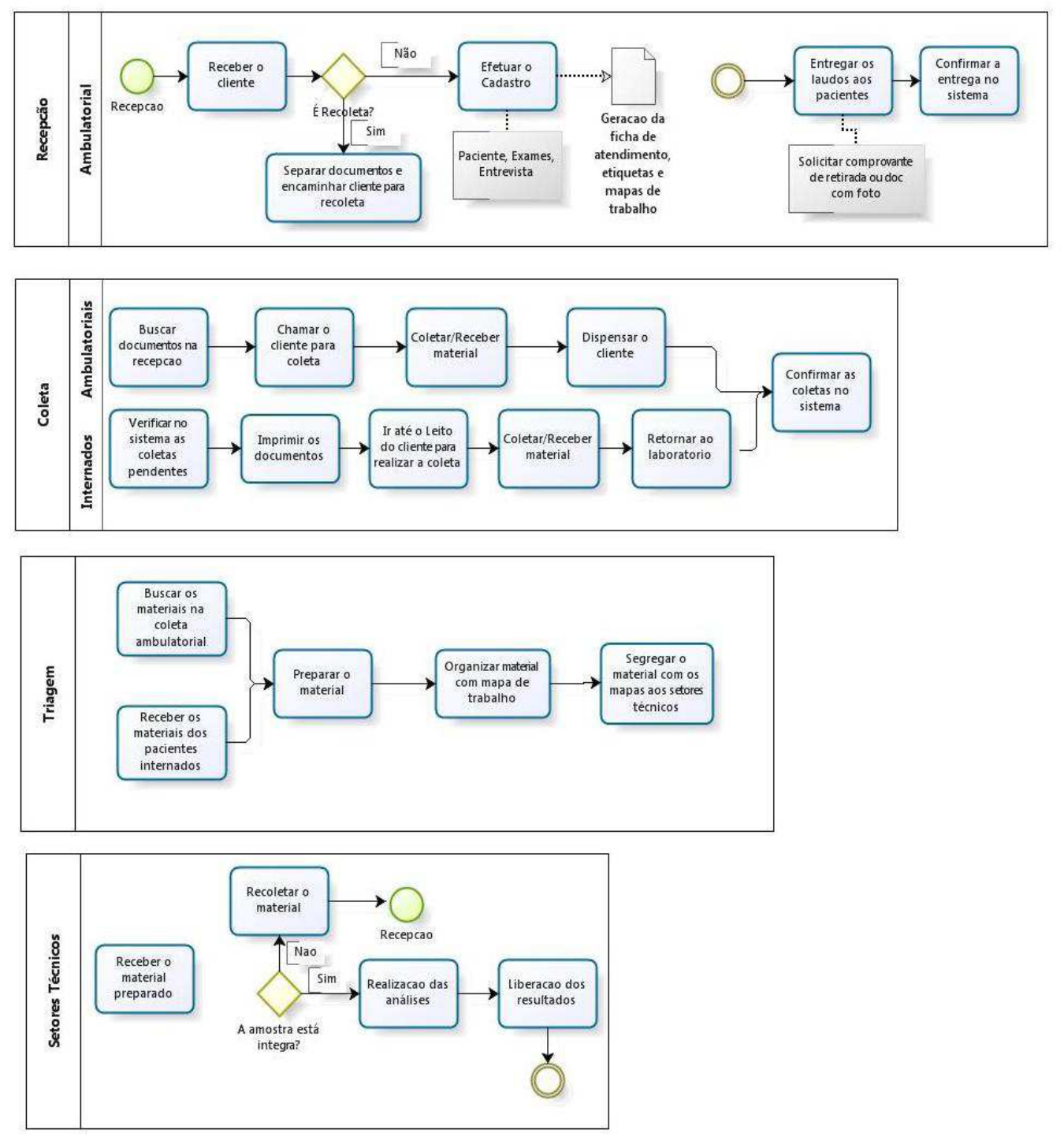

Fonte: Elaborado pelos autores

Revista Produção Online, Florianópolis, SC, v.12, n. 4, p. 1031-1056, out./dez. 2012. 
O início do processo ambulatorial no Laboratório se dá pelo atendimento ao cliente, momento em que é realizado o cadastro, inclusão dos exames e entrevista com o cliente diretamente no software de gestão utilizado pela Instituição, função realizada pelas Recepcionistas. Neste momento também são recebidos alguns materiais colhidos pelo paciente. Ao finalizar o cadastro, são impressos os mapas de trabalho e etiquetas de códigos de barras e é entregue ao cliente o comprovante de retirada dos exames.

O cliente é então encaminhado para a coleta de sangue. A Coletadora busca os mapas de trabalho e etiquetas na recepção e chama pelo cliente para realizar a coleta do material necessário para a realização dos exames solicitados pelo médico assistente. Após a coleta do material, o cliente é liberado e a Coletadora confirma a coleta do material no sistema, com o intuito de garantir a rastreabilidade das amostras. A cada hora são recolhidos, por uma auxiliar de laboratório, os materiais já colhidos dos pacientes ambulatoriais e são encaminhados ao setor de triagem.

O processo para atendimento de pacientes hospitalizados possui algumas diferenças do atendimento ambulatorial, ou seja, o médico assistente solicita dos exames para o paciente eletronicamente, escolhendo data e horário previsto para realização dos exames. Conforme horário previsto para execução dos exames, são impressos os mapas de trabalho e etiquetas para a coleta. A Coletadora vai até o paciente realizar a coleta e ao retornar confirma as coletas no sistema e encaminha o material para triagem.

Todos os materiais colhidos e recebidos dos pacientes (internados e ambulatoriais) são enviados ao setor de triagem de materiais juntamente com seus mapas de trabalho. Os materiais são preparados conforme identificação constante na etiqueta de código de barras, organizados com seu respectivo mapa de trabalho, de acordo com a prioridade de atendimento e enviados aos setores técnicos. As atividades de preparo e de segregação dos materiais são realizadas pelas Auxiliares de Laboratório.

Ao chegar ao Setor Técnico, os materiais são analisados criticamente aplicando-se critérios de aceitação e rejeição pré-estabelecidos e descritos no procedimento operacional padrão. Depois de aceitos, os materiais são processados, anotando-se os resultados nos respectivos mapas de trabalho para posterior 
conferência eletrônica, ou seja, liberação dos resultados para apreciação do médico assistente.

Cabe ressaltar que, quando necessário, são solicitadas recoletas dos materiais rejeitados pelo Setor Técnico. Para isso, são indicados, no sistema de gestão, os exames que devem ser recoletados, informando, obrigatoriamente, 0 motivo da recoleta. Na sequência, o mapa de trabalho é encaminhado à respectiva recepção, de acordo com o atendimento (seja ambulatorial ou proveniente de internação), para realizar o contato com o paciente. As atividades realizadas nos Setores Técnicos são desempenhadas pelos Farmacêuticos-Bioquímicos.

\subsection{Identificação de Oportunidade de Melhorias}

Após a realização do mapeamento dos macroprocessos, pode-se observar e analisar a ocorrências dos problemas nos processos internos do Laboratório, com o objetivo de identificar oportunidades de melhorias. Já no início do processo de atendimento aos clientes, observou-se que o processo não garante a identificação do paciente no momento da coleta das amostras, uma vez que o documento de identificação com foto só é solicitado no cadastro. Dessa forma, pode-se reorganizar o processo de atendimento, de tal forma que o processo assegure a identificação de todos os clientes. Ainda no atendimento ao cliente, o cliente recebe o comprovante de retirada dos exames ao finalizar o cadastro. Este comprovante é o documento legal para que o paciente ou outrem retire os resultados dos exames realizados. Dessa forma, este procedimento não garante que o mesmo realizará a coleta do material para posterior retirada dos resultados.

Outro aspecto relevante no atendimento é que se observa frequentemente a geração de filas e, consequentemente, demasiado tempo de espera, para o cadastro e coleta de amostras. As filas se desenvolvem devido ao excesso de atividades em cada processo (cadastro e coleta), ou seja, no cadastro, além de efetuar a inclusão dos dados do paciente deve-se aguardar a impressão de todos os documentos necessários para a realização dos exames do paciente. $\mathrm{Na}$ coleta de material, as filas se formam, pois a Coletadora precisa ir até a Recepção buscar os documentos 
para efetuar a coleta do sangue e após efetuar a coleta deve separar estes documentos conforme os setores para os quais os materiais serão enviados.

Pôde-se constatar, também, que há um grande fluxo de documentos, ou seja, os mapas de trabalho, as etiquetas de códigos de barras, as fichas de atendimento e as requisições de exames passam por diversas etapas antes de serem utilizados nos setores técnicos, ocasionando a perda de alguns documentos antes de chegarem ao seu destino. O extravio destes documentos implica no prazo de entrega dos exames, como também há a possibilidade de não realização do exame em tempo hábil, necessitando que o paciente retorne ao laboratório para repetir o exame.

Outro aspecto importante observado nos processos avaliados é a inexistência de uma forma clara para diferenciação dos documentos de pacientes ambulatoriais e hospitalizados, ou seja, não é possível identificar a distância quais amostras são urgentes e devem ser analisadas e liberadas primeiramente.

No Setor de Triagem, observou-se que o material fica aguardando o processo de centrifugação por falta de espaço nas centrifugas existentes. Com as centrífugas existentes no laboratório é possível centrifugar 60 tubos a cada 25 minutos. Por conseguinte, verifica-se a presença de um gargalo no processo, ou seja, o processo de centrifugação restringe e determina o ritmo e a capacidade produtiva. Nos Setores Técnicos, por sua vez, onde todo o material é processado, contatou-se que há somente o sistema de registro das solicitações de recoletas, não existindo um sistema de monitoramento e análise dos dados registrados.

\subsection{Proposta de Reestruturação dos Processos Analisados}

Após o mapeamento e o diagnóstico dos processos, foi possível elaborar uma proposta de reestruturação dos macroprocessos existentes. Para qualificar os processos e agilizar o atendimento aos clientes, a Instituição precisa descentralizar as atividades e reorganizar os fluxos no atendimento, pois a demora no atendimento é consequência de um processo burocrático.

Sendo assim, foi proposta a descentralização das impressões dos mapas de trabalho, etiquetas de código de barras, ficha de atendimento e requisição de 
exames, ou seja, os mapas devem ser impressos diretamente no respectivo setor técnico e as etiquetas e o comprovante de retirada devem ser impressos na sala de coletas, onde a ordem de impressão corresponde à ordem de chamada para a coleta e a ficha de atendimento e requisição de exames devem ser impressas na recepção para o acompanhamento do faturamento destes exames. Dessa forma, todos os documentos seriam emitidos nos setores em que serão utilizados, evitando que se percam, reduzindo o tempo entre os processos, otimizando o atendimento do cliente, o processamento das amostras e entrega dos resultados.

Com a reorganização do fluxo de atendimento, passa-se a garantir a identificação do paciente no momento da coleta, já que será solicitado, neste momento, um documento com a sua imagem (fotografia). Ainda com a finalidade de assegurar a sequência correta do atendimento, o comprovante de retirada de exames será entrega somente após a coleta do material, impossibilitando o paciente de vir requisitar os resultados dos exames sem ter coletado ou entregue o respectivo material para análise.

Os prazos de entrega de resultados são diferenciados para urgências ambulatoriais e para pacientes internados. Estes prazos foram previamente estabelecidos em comum acordo com a equipe médica. O Laboratório precisa evidenciar estes mapas de trabalho que contem exames com prazo diferenciado para garantir o cumprimento, em sua totalidade, destes prazos. Acredita-se que a diferenciação por cores dos mapas de trabalho seja mais eficiente que a informação da procedência no mapa de trabalho. Dessa maneira, seria possível diferenciar os mapas de trabalho por tipo de atendimento, ou seja, uma cor para atendimento de paciente ambulatorial e outra cor para atendimento de paciente hospitalizado (internação). Seria interessante, ainda, utilizar cores diferenciadoras nos materiais e mapas de pacientes com exames urgentes. Além disso, sugere-se que a informação sobre o caráter do atendimento (urgência ou rotina) seja bem visível para ser prontamente identificada pelos profissionais.

A demora no preparo do material para as análises ocorre em virtude do maior volume de análises serem solicitadas e realizadas pela manhã, em função das orientações com relação ao preparo do paciente. Entretanto, foi constatado que um dos motivos que contribui para esta demora é a quantidade/capacidade de 
centrífugas, pois o laboratório possui apenas três centrífugas para processar todo o material, onde são centrifugados apenas 60 tubos por vez. Tendo em vista que $80 \%$ do material dos pacientes passa pelo processo de centrifugação por, no mínimo, 25 minutos, antes de ser analisado, deve-se aumentar o número de centrífugas ou a capacidade das centrífugas para agilizar o processo de centrifugação e evitar que o material fique aguardando para ser processado.

O sistema de registro dos exames que necessitam ser recoletados, no software de gestão existente, é muito eficiente, porém, não são realizadas análises destes dados pela equipe do Laboratório. Assim, foi sugerida a implantação de um sistema eficaz para o monitoramento dos motivos de recoletas no sentido de reduzir ao máximo o índice de recoletas e melhorar continuamente o processo relacionado. É importante ressaltar que as recoletas representam retrabalho, ou seja, repetição do procedimento, desperdiçando tempo e recursos, devendo ser, portanto, reduzidas ao limite ou eliminadas do processo.

Em acréscimo, foi recomendada a elaboração de um cronograma de atividades de capacitação e aperfeiçoamento para todos os colaboradores do Laboratório, observando-se seu perfil, sua atuação e necessidades, com o objetivo de orientá-los em relação aos procedimentos e cuidados que devem ser tomados ao realizar a coleta de sangue, a fim de minimizar os erros e rejeições de materiais inadequados.

De acordo com a Figura 2, observa-se o mapa de contexto proposto, o qual contempla as propostas para a reestruturação dos macroprocessos analisados. 
Figura 2 - Mapa de contexto proposto para os macroprocessos do Laboratório

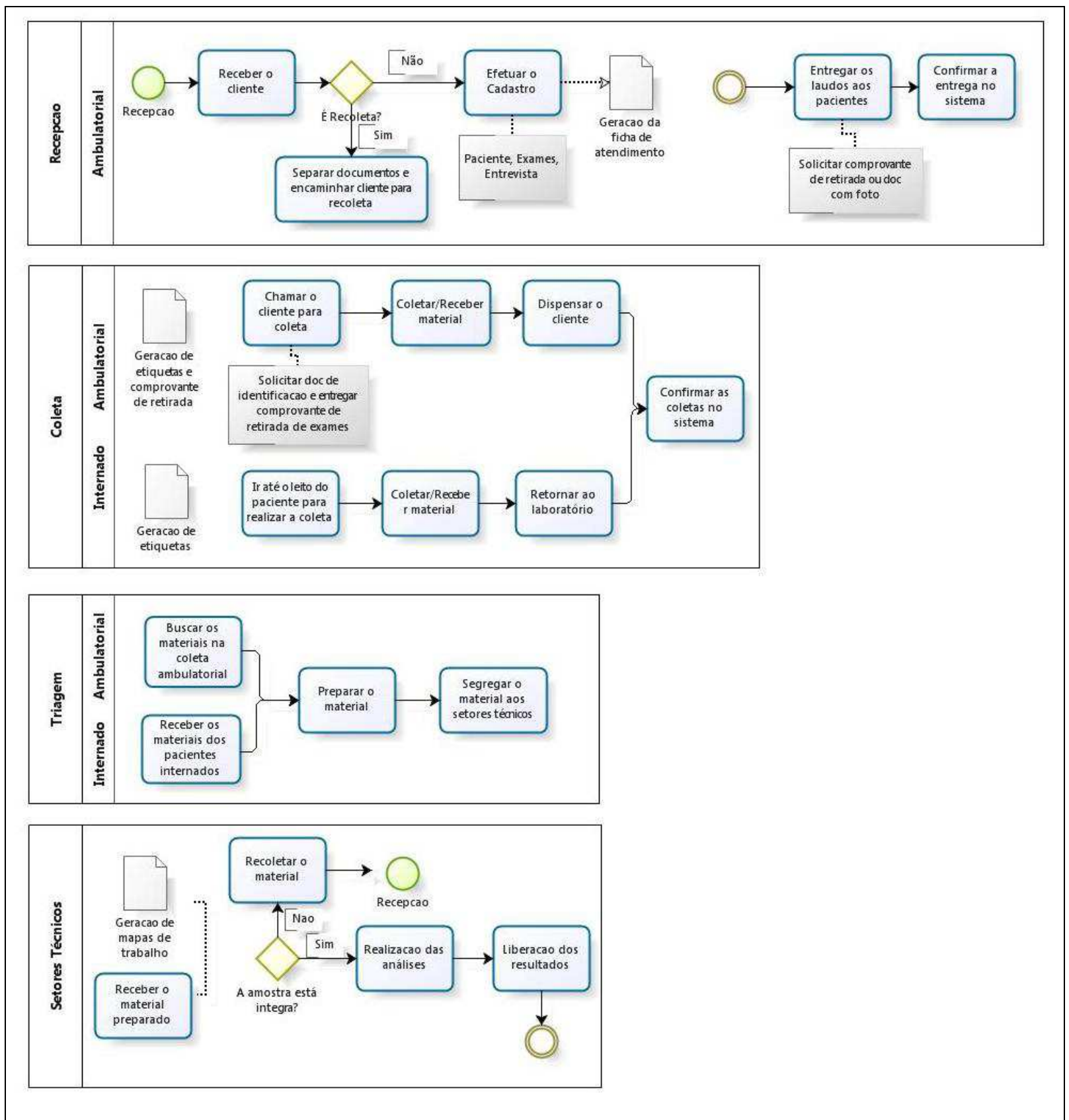

Fonte: Elaborado pelos autores

\subsection{Indicadores de Desempenho e Metas}

A análise criteriosa dos macroprocessos do Laboratório possibilitou a identificação e a sugestão de indicadores de desempenho, de forma a permitir uma forma de gestão que contemple as necessidades de controle e as definições estratégicas estabelecidas pela alta administração da Instituição.

Revista Produção Online, Florianópolis, SC, v.12, n. 4, p. 1031-1056, out./dez. 2012. 
Alguns dos indicadores enumerados foram identificados pelos próprios colaboradores, que se mostraram receptivos ao acompanhamento do desempenho dos processos por meio de indicadores para facilitar a gestão e possibilitar informações oportunas para melhorias contínuas. Ressalta-se que alguns processos não apresentavam indicadores de desempenho e metas e, em outros, não eram, de fato, consistentes.

O mapeamento detalhado do processo facilitou a definição dos indicadores, pois forneceu dados sobre as atividades e sobre os documentos de registro já existentes no processo. Assim sendo, a origem dos dados para apuração dos indicadores está na base de dados atual e a frequência recomendada para a apuração, divulgação e análise dos indicadores é mensal, exceto para aqueles indicadores que embasam o processo de tomada de decisões dos gestores, de forma cotidiana. Entretanto, as metas constituem uma novidade para o Laboratório, uma vez que, até então, não trabalhava com qualquer sistema de avaliação de desempenho.

Para que os resultados provenientes da implantação da reestruturação possam ser efetivamente acompanhados, gerando informações úteis à tomada de decisões, foram sugeridos alguns indicadores de desempenho, acompanhados de suas respectivas metas, os quais foram desenvolvidos junto com o corpo de profissionais (vide Quadro 1). 
Quadro 1 - Indicadores de desempenho e metas propostos

\begin{tabular}{|c|c|c|c|}
\hline Indicadores & $\begin{array}{l}\text { Composição } \\
\text { do Indicador }\end{array}$ & Objetivo & Metas \\
\hline $\begin{array}{l}\text { Tempo total de } \\
\text { atendimento - } \\
\text { urgência ambulatorial }\end{array}$ & $\begin{array}{l}\text { Data/hora da aprovação } \\
\text { dos exames - data/hora } \\
\text { prevista }\end{array}$ & $\begin{array}{l}\text { Monitorar o } \\
\text { cumprimento dos prazos } \\
\text { para entrega dos } \\
\text { resultados }\end{array}$ & $\begin{array}{l}90 \% \text { dos } \\
\text { exames } \\
\text { urgentes } \\
\text { ambulatoriais } \\
\text { entregues com } \\
\text { prazo de } 8 \\
\text { horas }\end{array}$ \\
\hline $\begin{array}{l}\text { Tempo total de } \\
\text { atendimento - } \\
\text { urgência internação }\end{array}$ & $\begin{array}{l}\text { Data/hora da aprovação } \\
\text { dos exames - data/hora } \\
\text { prevista }\end{array}$ & $\begin{array}{l}\text { Monitorar o } \\
\text { cumprimento dos prazos } \\
\text { para entrega dos } \\
\text { resultados }\end{array}$ & $\begin{array}{l}90 \% \text { dos } \\
\text { exames } \\
\text { urgentes } \\
\text { entregues com } \\
\text { prazo de } 2 \\
\text { horas }\end{array}$ \\
\hline $\begin{array}{l}\text { Tempo entre } \\
\text { atendimento de coleta - } \\
\text { atendimento } \\
\text { ambulatorial } \\
\text { preferencial }\end{array}$ & $\begin{array}{l}\text { Data/hora coleta - } \\
\text { data/hora início do } \\
\text { atendimento }\end{array}$ & $\begin{array}{l}\text { Monitorar o tempo de } \\
\text { atendimento } \\
\text { ambulatorial preferencial }\end{array}$ & $\begin{array}{l}90 \% \text { dos } \\
\text { pacientes } \\
\text { atendidos com } \\
\text { tempo inferior a } \\
15 \text { minutos }\end{array}$ \\
\hline $\begin{array}{l}\text { Tempo entre } \\
\text { atendimento de coleta - } \\
\text { atendimento } \\
\text { ambulatorial normal }\end{array}$ & $\begin{array}{l}\text { Data/hora coleta - } \\
\text { data/hora início do } \\
\text { atendimento }\end{array}$ & $\begin{array}{l}\text { Monitorar o tempo de } \\
\text { atendimento } \\
\text { ambulatorial normal }\end{array}$ & $\begin{array}{l}90 \% \text { dos } \\
\text { pacientes } \\
\text { atendidos com } \\
\text { tempo inferior a } \\
30 \text { minutos } \\
\end{array}$ \\
\hline$\%$ de recoletas & $\begin{array}{l}\text { (Número de } \\
\text { recoletas/número de } \\
\text { exames realizados) } \times 100\end{array}$ & $\begin{array}{l}\text { Monitorar a quantidade } \\
\text { de exames recoletados }\end{array}$ & $<1 \%$ \\
\hline$\%$ de laudos corrigidos & $\begin{array}{l}\text { (Número de laudos } \\
\text { corrigidos/número de } \\
\text { atendimentos) x } 100\end{array}$ & $\begin{array}{l}\text { Monitorar a quantidade } \\
\text { de laudos emitidos com } \\
\text { erros }\end{array}$ & $0 \%$ \\
\hline$\%$ perda de insumos & $\begin{array}{l}\text { [1- (amostras } \\
\text { processadas/testes } \\
\text { consumidos)] x } 100\end{array}$ & $\begin{array}{l}\text { Monitorar a utilização de } \\
\text { insumos com outras } \\
\text { finalidades que não } \\
\text { processar amostras de } \\
\text { pacientes }\end{array}$ & $<10 \%$ \\
\hline Glosas & $\begin{array}{l}\text { Número de } \\
\text { procedimentos não pagos }\end{array}$ & $\begin{array}{l}\text { Monitorar o processo de } \\
\text { faturamento, como } \\
\text { também todo o } \\
\text { processo laboratorial. }\end{array}$ & Nenhuma \\
\hline
\end{tabular}

Fonte: Elaborado pelos autores

\subsection{Recursos Necessários à Implementação da Reestruturação Proposta}

Para a reestruturação dos processos analisados, serão necessários alguns investimentos, dentre eles a aquisição de três impressoras de etiquetas de código de barras, onde duas deverão ser instaladas na sala de coletas ambulatoriais, uma para 
atendimento normal e outra para o atendimento preferencial, que indicarão a ordem de chamada dos pacientes para coleta.

A outra impressora será instalada na Secretaria interna do Laboratório para atender aos pacientes internados no Hospital. Além disso, será preciso ampliar o número de impressoras a laser para impressão dos mapas de trabalho. $O$ Laboratório possui quatro setores (Bioquímica, Hematologia, Imunologia/Laboratório de Apoio e Microbiologia/ Uroanálise/Parasitologia) e conforme, proposto, cada setor deverá possuir uma impressora.

Durante o desenvolvimento do trabalho também foi identificada a presença de gargalo no processo de centrifugação, onde as amostras ficam aguardando serem processadas. Dessa forma, a aquisição de, no mínimo, três centrífugas, aumentaria em $50 \%$ o volume de amostras centrifugadas a cada 25 minutos, podendo ainda ser direcionada uma centrífuga para amostras urgentes, o que eliminaria o tempo em que as amostras permanecem paradas e, por consequência, diminuiria o tempo de entrega de resultados destes exames.

A Tabela 1 apresenta o resumo dos recursos que seriam necessários à implementação da reestruturação proposta.

Tabela 1 - Recursos necessários à implementação da reestruturação proposta

\begin{tabular}{lccc}
\hline \multicolumn{1}{c}{ Equipamentos Necessários } & Quantidades & $\begin{array}{c}\text { Valor Unitário } \\
\text { (R\$) }\end{array}$ & $\begin{array}{c}\text { Valor Total } \\
\text { (R\$) }\end{array}$ \\
\hline $\begin{array}{l}\text { Impressora de etiquetas de código } \\
\text { de barras }\end{array}$ & 03 & 800,00 & $2.400,00$ \\
\hline Impressora a laser T640 & 04 & $2.100,00$ & $8.400,00$ \\
\hline $\begin{array}{l}\text { Centrífuga Combate } \\
\text { (capacidade de 28 tubos) }\end{array}$ & 03 & $3.800,00$ & $11.400,00$ \\
\hline \multicolumn{1}{c}{ Investimento Total Necessário (R\$) } & & $22.000,00$ \\
\hline
\end{tabular}

Fonte: Elaborada pelos autores

\section{CONSIDERAÇÕES FINAIS}

O mapeamento de processos está sendo empregado por organizações, tanto de manufatura quanto de serviços, na implementação de seus sistemas de gestão da qualidade, visando identificar, definir, medir e analisar os processos com o objetivo de alcançar melhorias contínuas, maior competitividade e um maior desempenho organizacional.

Revista Produção Online, Florianópolis, SC, v.12, n. 4, p. 1031-1056, out./dez. 2012. 
No caso das organizações prestadoras de serviços, mais especificamente da área da saúde, a aplicação da gestão por processos, e do mapeamento dos mesmos, tem um apelo ainda maior em virtude das características inerentes a este tipo de processo, como é o caso da intangibilidade dos serviços, o alto nível de incerteza e as relações de interação com o cliente que, em muitos casos, participa do processo de entrega do serviço.

Aliada ao mapeamento dos processos, o levantamento de informações auxiliares torna a análise dos processos mais precisa e próxima da realidade. No caso estudado, as informações pertinentes aos processos de atendimento ao cliente e coleta de materiais, como tempo de espera para coleta e tempo total de atendimento são importantes, pois estão diretamente ligadas ao cliente, representando uma medida de sua satisfação direta, facilitando ao máximo a determinação do valor percebido pelo cliente, isto é, fornecendo a base para se determinar quais, dentre as atividades executadas, estão de acordo com as exigências do cliente e, dessa forma são de valor agregado, e quais são executadas por motivos diversos da satisfação do cliente e, portanto, não agregam valor.

O mapeamento de processos gerou uma facilidade na observação dos processos, propiciando uma análise detalhada com o objetivo de melhorar 0 processo, identificando as atividades que não agregam valor, simplificando as atividades de importância capital na gestão por processos.

As alterações sugeridas para a reestruturação dos processos analisados no Laboratório diminuem o tempo de processamento da operação-gargalo, e esta mudança acarretaria ganhos representativos na quantidade produzida. Quanto menor o tempo em que as amostras permanecerem paradas, melhor a qualidade do material a ser analisado e mais fidedigno o resultado. E tal modificação também impactará na qualidade do serviço prestado.

Por meio da utilização do Blueprint (representação do mapa de contexto) foi possível identificar e eliminar atividades que não agregavam valor ao paciente, salientando que pode ser utilizado como base científica para a tomada de decisões sem interferir no sistema real, uma vez que os benefícios para a empresa e seus clientes serão evidentes. Esta reestruturação é um primeiro passo a ser dado para a melhoria dos principais processos do laboratório, por seu baixo custo de 
investimento e a simplicidade da solução. Assim, transforma-se o processo existente em um processo com maior capacidade de produção e menor tempo total de atendimento, obtendo-se um melhor desempenho nos serviços realizados e maior satisfação aos clientes atendidos.

Nesta direção, as alterações propostas permitirão negociar a ampliação do rol de procedimentos e exames oferecidos aos clientes da rede pública, além de rever e melhorar as metas contratualizadas com o SUS. Consequentemente, possibilitará a atender um número maior de clientes e com qualidade superior, mantendo sua estrutura e custos operacionais, resultando em um desempenho organizacional mais expressivo.

É importante comentar que o laboratório clínico tem um papel essencial no sistema de saúde. A maioria das decisões médicas é tomada utilizando as informações fornecidas pelos processos laboratoriais. Gerenciar adequadamente estes processos é vital para a segurança do paciente. Um sistema de indicadores bem definido, padronizada e constantemente monitorado é o maior aliado nesse desafio diário que é gerenciar processos em um laboratório.

Entender e atender as necessidades e as expectativas dos clientes é o principal passo para uma posição diferenciada em termos de competitividade no mercado. Isso não é diferente para o laboratório clínico, onde se vivencia um momento de intensa consolidação e elevada competitividade, em que atender às expectativas dos clientes e gerenciar os processos críticos é essencial. Melhorar continuamente os processos não é um privilégio, mas sim uma necessidade. Isso porque as necessidades dos clientes mudam continuamente e, caso não haja agilidade e flexibilidade nos processos para atender a esta mudança contínua de requisitos, haverá perda de competitividade e, o mais importante, o não cumprimento do papel da Instituição em sua plenitude.

O estudo realizado, portanto, demonstrou a relevância do mapeamento dos processos, uma vez que poderá gerar benefícios diretos e indiretos para a organização. Dentre eles, destaca-se um maior conhecimento dos processos realizados, a fim monitorá-los por meio dos indicadores propostos, que podem vir a contribuir diretamente para uma melhor performance. Observa-se, por fim, uma perspectiva de geração no longo prazo de uma maior eficácia na realização das 
atividades da empresa, consolidando os benefícios que o mapeamento de processos e seus possíveis desdobramentos podem trazer a toda organização.

\section{REFERÊNCIAS}

BRANDÃO, C. R. Pesquisa participante. 8. ed. São Paulo: Brasiliense, 1990.

BROWN, S. W.; FISK, R. P.; BITNER, M. J. The development and emergence of service marketing thought. International Journal of Service Industry Management, v. 5, n. 1, p. 21-48, 1994.

CATELLI, A. Controladoria: uma abordagem da gestão econômica. 2. ed. São Paulo: Atlas, 2001.

CAMPOS, V. F. TQC: controle da qualidade total (no estilo japonês). 6. ed. Belo Horizonte: UFMG, 1995.

DAVENPORT, T. H. Reengenharia de processos: como inovar na empresa através da tecnologia da informação. 5. ed. Rio de Janeiro: Campus, 1995.

FITZSIMMONS, J. A.; FITZSIMMONS, M. Administração de serviços: operações, estratégia e tecnologia de informação. 6. ed. Porto Alegre: Bookman, 2010.

GIANESI, I. G. N.; CORRÊA, H. L. Administração estratégica de serviços: operações para a satisfação do cliente. São Paulo: Atlas, 1994.

GIL, A. C. Métodos e técnicas da pesquisa social. 6. ed. São Paulo: Atlas, 2008.

GONÇALVES, J. E. L. Processo, que processo? Revista de Administração de Empresas (RAE), v. 40, n. 4, p. 8-19, 2000.

GUMMESSON, E. Service management: an evaluation and the future. International Journal of Service Industry Management, v. 5, n. 1, p. 77-96, 1994.

HAMMER, M.; CHAMPY, J. Reengenharia: revolucionando a empresa em função dos clientes, da concorrência e das grandes mudanças da gerência. 28. ed. Rio de Janeiro: Campus, 1995.

HARRINGTON, J. Aperfeiçoando processos empresariais. São Paulo: Makron Books, 1993.

HRONEC, S. M. Sinais vitais. São Paulo: Makron Books, 1994.

HUNT, V. D. Process mapping: how to reengineer your business process. New York: John Wiley \& Sons, 1996. 
JOHANSSON, H. J.; MCHUGH, P.; PENDLEBURY, A. J.; WHEELER III, W. A. Processos de negócios: como criar sinergia entre a estratégia de mercado e a excelência operacional. São Paulo: Pioneira, 1995.

JOHNSTON, R.; CLARK, G. Administração de operações de serviço. São Paulo: Atlas, 2002.

LOVELOCK, C. H. Product plus: produto + serviço = vantagem competitiva. São Paulo: Makron Books, 1995.

MARTINS, R. A.; NETO, P. L. O. C. Indicadores de desempenho para gestão pela qualidade total: uma proposta para sistematização. Gestão \& Produção, v. 5, n. 3, p. 298-311, 1998.

MELLO, C. H. P.; SILVA, C. E. S.; TURRIONI, J. B.; SOUZA, L. G. M. ISO 9001:2000 - sistema de gestão da qualidade para operações de produção e serviço. São Paulo: Atlas, 2002.

MILAN, G. S.; TREZ, G. Pesquisa de satisfação: um modelo para planos de saúde. RAE-eletrônica, v. 4, n. 2, p. 1-21, jul./dez. 2005.

RAMASWAMY, R. Design and management of service processes: keeping customers for life. New York: Addison-Wesley, 1996.

RICHARDSON, R. J. Pesquisa social: métodos e técnicas. 3. ed. São Paulo: Atlas, 1999.

SANTOS, L. C.; VARVAKIS, G. Projeto e análise de processos em serviços: uma avaliação de técnicas de representação. Produto \& Produção, v. 5, n. 3, p. 1-16, 2001.

SCHMENNER, R. W. Administração de operações em serviços. São Paulo: Futura, 1999.

SHOSTACK, G. L. Designing services that deliver. Harvard Business Review, v. 62 , n. 1, p. 133-139, 1984.

SILVA, R. F. Indicadores de eficiência e eficácia: uma visão prática sobre indicadores de desempenho para avaliar a eficiência e a eficácia dos processos organizacionais. Qualypro, p.1-11, 2007. Disponível em:

<http://www.qualypro.com.br/novosite/outros_artigos.asp?id=27>. Acesso em: 26 set. 2010.

SINK, D. S.; TUTTLE, T. C. Planejamento e medição para a performance. Rio de Janeiro: Qualitymark, 1993.

TAKASHINA, N. T.; FLORES, M. C. X. Indicadores da qualidade e do desempenho: como estabelecer metas e medir resultados. Rio de Janeiro: Qualitymark, 1996. 
TORRES JÚNIOR, N.; MIYAKE, D. I. Melhoria de desempenho em serviços: alternativas para lidar com o trade-off entre eficiência e eficácia. Revista Produção Online, v. 11, n. 1, p. 162-193, mar. 2011.

VILLELA, C. S. S. Mapeamento de processos como ferramenta de reestruturação e aprendizado organizacional. Dissertação (Mestrado)- Programa de Pós-Graduação em Engenharia de Produção, Universidade Federal de Santa Catarina. Florianópolis, 2000.

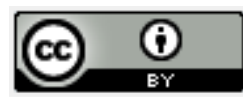

Artigo recebido em 11/05/2011 e aceito para publicação em 18/09/2011. 